\title{
Une étude sur la supériorité des blés hybrides au niveau des capacités de remplissage du grain : résultats d'une expérimentation multilocale
}

\author{
FX Oury ${ }^{1 *}$, P Brabant 2, P Pluchard 3, P Bérard 1, M Rousset 1 \\ avec la collaboration technique de J Gourdon 1, F Lagoutte 1, H Corti 2, N Galic 2, A Blake ${ }^{3}$ \\ ${ }^{1}$ INRA, Station d'amélioration des plantes, domaine de Crovelle, F63039 Clermont-Ferrand, Cedex \\ 2 Station de génétique végétale, ferme du Moulon, F91120 Gif-sur-Yvette; \\ 3 INRA, domaine de Brunehaut, Estrées-en-Chaussées, F80200 Péronne
}

(Reçu le 7 décembre 1992; accepté le 15 février 1993)

\begin{abstract}
Résumé - Une expérimentation multilocale nous a permis de comparer 29 hybrides de blé tendre d'hiver avec leurs parents, dans des conditions environnementales variées. Ce matériel a été évalué dans des dispositifs en blocs complets randomisés, la parcelle élémentaire ayant au moins $6 \mathrm{~m}^{2}$. Dans les 3 lieux, il y a un hétérosis par rapport au parent supérieur pour la matière sèche aérienne à la récolte. Cette production de biomasse plus importante est associée à un hétérosis pour le rendement en grains, en raison d'une assimilation post-floraison plus importante chez la $F_{1}$ : l'indice de récolte des hybrides reste ainsi équivalent à celui des lignées. La $F_{1}$ ne manifeste pas de supériorité pour la fertilité, mais elle se distingue de ses parents par des capacités de remplissage plus importantes. Celles-ci sont dues au fait que l'hybride présente une vitesse de remplissage des grains plus élevée que celle de ses parents, pour une durée de remplissage au moins équivalente. La supériorité de l'hybride au niveau de la vitesse de remplissage apparaît liée à la disponibilité en assimilats au moment de la floraison, qui est plus importante pour la $F_{1}$ en raison d'un hétérosis pour la matière sèche des tiges $10 \mathrm{j}$ après la floraison. Plus généralement, la situation hybride paraît caractérisée par une relation «source-puits» plus favorable que celle existant chez la lignée.
\end{abstract}

blé hybride / hétérosis / remplissage du grain / composante du rendement

Summary - The superiority of wheat hybrids for grain filling: results of a multilocal experiment. In a multilocal experiment, 29 winter wheat hybrids and their parents were compared under various environmental conditions. The material was evaluated in randomised complete block designs, the elementary plot measuring $6 \mathrm{~m}^{2}$ minimum. At the 3 locations, heterosis over the best parental line was demonstrated for biomass production. This higher biomass production was related to heterosis over the best parental line for grain yield, because of a higher post-anthesis assimilation for F1: so the harvest index of the hybrids remained equivalent to that of the parent lines. Fertility was not greater for $F 1$ than for inbred lines, but the grain filling capacity of hybrids was greater than that of parental lines. Hybrids had a higher grain filling rate than parental lines for an equivalent duration of grain filling. Hybrid superiority for filling rate appeared to be related to assimilates availability at anthesis, which was greater for $F 1$ (due to heterosis for stem dry matter 10 days after anthesis). On the whole, hybrids seemed to have a more favourable "source-sink" relation than inbred lines.

hybrid wheat / heterosis / grain filling / yield component

* Correspondance et tirés à part 


\section{INTRODUCTION}

La production de blés hybrides par voie chimique permet de disposer de quantités de semences suffisantes pour de nombreuses combinaisons $F_{1}$. Grâce à cette technique, il est possible d'étudier l'élaboration du rendement des hybrides dans des conditions de peuplement proches de celles de la pratique agricole, et sur des effectifs suffisamment importants pour que les résultats aient une valeur statistique. Deux expérimentations (Oury et al, 1990a,b), portant sur 2 lots de génotypes différents (respectivement 34 et $35 F_{1}$, comparées à leurs parents), avaient permis de conclure que les hybrides ne se distinguaient pas des lignées du point de vue de la fertilité (celle-ci correspondant au nombre de grains produits par $\mathrm{m}^{2}$ ). En particulier, pour un même nombre d'épis par $\mathrm{m}^{2}$, on n'obtenait pas un nombre de grains par épi plus élevé chez l'hybride que chez la lignée. Par contre, il apparaissait que les hybrides avaient des capacités de remplissage supérieures à celles des lignées: pour un même nombre de grains par $\mathrm{m}^{2}$, l'hybride obtenait un poids de 1000 grains plus élevé que la lignée.

Cet hétérosis pour le poids de 1000 grains a été trouvé par de nombreux auteurs travaillant sur le blé (Goujon et Paquet, 1968; Keydel, 1973; Borghi et al, 1988; Morgan et al, 1989). II nous a paru intéressant d'analyser plus finement ce qui se passait au cours de la période "floraison-maturité", pendant laquelle s'effectue le remplissage du grain. L'objectif était de rechercher les mécanismes à la base de l'hétérosis pour le poids de 1000 grains. Pour cela, nous avons étudié la production de biomasse à la floraison et sa réallocation vers le grain, ainsi que l'assimilation post-floraison et la cinétique de remplissage du grain. Cet article présente les résultats d'une expérimentation multilocale, mise en place dans le cadre de la collaboration entre I'INRA et le GIE Hybriblé. Les essais ont été conduits pendant la campagne 1990-1991, sur les stations INRA de Clermont-Ferrand et Monsen-Chaussée, ainsi qu'à la station de génétique végétale du Moulon. Ces essais nous ont permis d'étudier l'élaboration du rendement chez des hybrides et leurs parents, en mettant plus particulièrement l'accent sur les vitesses et les durées de remplissage du grain.

\section{MATÉRIEL ET MÉTHODES}

Nous avons mis en expérimentation 40 hybrides produits avec l'agent chimique d'hybridation d'Orsan, à partir de lignées fournies par les différents membres du GIE Hybriblé et par les différentes stations INRA partenaires de ce GIE. La pureté de 11 de ces 40 hybrides, estimée visuellement, nous a paru insuffisante (plus de $20 \%$ de plantes de type femelle ou hors type), et nous avons donc retenu $29 \mathrm{~F}_{1}$ pour notre étude. Ces $29 F_{1}$ faisaient intervenir 15 parents femelles et 8 parents mâles (ces 23 lignées parentales, d'origines très diverses, représentaient une gamme de variabilité génétique importante), sans plan de croisement particulier. Un des 29 hybrides n'a pu être évalué pour le rendement au Moulon, et un autre était absent de l'essai de Mons. Deux des 23 parents n'ont pas pu être semés au Moulon. Pour ce lieu, les valeurs d'hétérosis par rapport aux parents moyen et supérieur n'ont donc pas pu être calculées pour les 3 hybrides dans lesquels ces 2 parents intervenaient.

Le dispositif expérimental commun aux 3 lieux comprenait 4 blocs complets randomisés. La parcelle élémentaire était constituée de 6 lignes de $5 \mathrm{~m}$ espacées de $20 \mathrm{~cm}$ à Clermont et à Mons, et de 6 lignes de $4 \mathrm{~m}$ espacées de $23 \mathrm{~cm}$ au Moulon. Les techniques culturales appliquées en chacun des lieux correspondaient aux conduites intensives habituellement pratiquées dans la région, avec en particulier des apports $N, P, K$ ajustés à des objectif́s de rendement élevés, et une couverture fongicide et insecticide efficace. L'essai de Mons a de plus reçu un traitement raccourcisseur.

Dans les 3 lieux, 2 des 4 répétitions ont servi à mesurer le rendement en grains à $15 \%$ d'humidité (RDT), ainsi que le poids de 1000 grains à $15 \%$ d'humidité (PMG). À Clermont et au Moulon ces 2 répétitions ont servi également à estimer le nombre d'épis par $\mathrm{m}^{2}$ (NE), par comptage des épis sur une longueur de $1 \mathrm{~m}$, sur 2 lignes de chacune des parcelles et en évitant les lignes de bordure. Le nombre de grains par $\mathrm{m}^{2}$ (NG) a ensuite été calculé à partir de $R D T$ et $P M G$, ainsi que le nombre de grains par épi (NGE), à partir de $N G$ et NE.

Les 2 autres répétitions ont permis la réalisation de 2 séries de prélèvements selon les protocoles suivants.

\section{Prélèvement de 3 échantillons de 100 tiges par parcelle}

Le premier échantillon est prélevé à la floraison, le deuxième $10 \mathrm{j}$ après la floraison, et le troisième à la récolte. Sur ces prélèvements nous avons mesuré la matière sèche (MS) aérienne, qui a été décomposée en "MS tiges", "MS balles + rachis" et "MS grains"

\footnotetext{
* Le GIE Hybriblé regroupe les établissements de sélection Blondeau, Momont, Orsem et UCASP, et la firme Orsan (pour la recherche sur les agents chimiques d'hybridation).
} 
(sauf pour le prélèvement réalisé à la floraison, pour lequel la MS des grains était encore nulle). À partir de la MS aérienne et de NE, nous avons calculé les productions de MS aérienne à l'hectare, à la floraison (MSf) et à la récolte (MSr). Le rapport entre "MS grains» et "MS aérienne», pour le prélèvement effectué à la récolte, a permis d'estimer l'indice de récolte (IR).

Nous avons calculé également, d'une part la quantité de MS provenant de l'assimilation post-floraison (obtenue par différence entre "MS aérienne" à la récolte et «MS aérienne» à la floraison), et d'autre part la quantité de MS de l'appareil végétatif remobilisée pour le remplissage des grains. Cette dernière est obtenue par différence entre "MS végétative" $10 \mathrm{j}$ après floraison et “MS végétative" à la récolte, la MS végétative correspondant à la "MS tiges + balles + rachis». En effet, la MS végétative augmente encore un peu après la floraison (fig 1), et c'est approximativement $10 \mathrm{j}$ après la floraison que les réserves carbonées sont maximales (Triboï et Ollier, 1991). À partir des valeurs obtenues sur les prélèvements et de NE, nous avons estimé la quantité de MS accumulée à l'hectare après floraison (ASS), ainsi que la quantité de MS remobilisée par hectare (REM). Ces estimations sont assez grossières, car elles reposent sur les hypothèses d'absence de pertes de MS après la floraison, par respiration ou par chute de feuilles, et de non-remobilisation d'assimilats à partir des racines (Gallagher et al, 1975; Le Gouis, 1992). Cependant, on peut noter que les pertes de MS après la floraison conduisent à une sous-estimation de ASS et une sur-estimation de
REM, alors que la remobilisation d'assimilats à partir des racines conduit à une sur-estimation de l'assimilation post-floraison et à une sous-estimation des remobilisations entre floraison et maturité.

\section{Prélèvement d'échantillons de 50 épis par parcelle}

Cinquante épis ont été prélevés tous les 5 à $7 \mathrm{j}$, à partir du $15^{\mathrm{e}}$ jour après la floraison et jusqu'à la maturité. Tous ces prélèvements (de 4 à 6 selon le génotype et le lieu) ont permis de suivre l'évolution de la MS de l'épi, qui a été décomposée en "MS grains» et “MS balles + rachis".

La figure 1 permet de visualiser, pour la variété «Renan» à Mons, les données issues des différents prélèvements, toutes les valeurs étant rapportées à 100 épis. Nous avons utilisé, pour exprimer le temps, une échelle thermique en ${ }^{\circ} \mathrm{C}$.j (base $0^{\circ} \mathrm{C}$ ). Toutes les mesures de MS ont été réalisées après passage à l'étuve pendant $48 \mathrm{~h}$ à $90^{\circ} \mathrm{C}$ (nous nous sommes assurés que cette durée d'étuvage était suffisante, en laissant certains échantillons plus longtemps et en vérifiant que les pesées effectuées au bout de $48 \mathrm{~h}$ donnaient le même résultat que celles réalisées après 72 ou 96 h).

Pour tous les prélèvements (excepté le premier), les grains obtenus après battage des épis ont permis d'estimer les poids de 1000 grains. Pour le prélève-

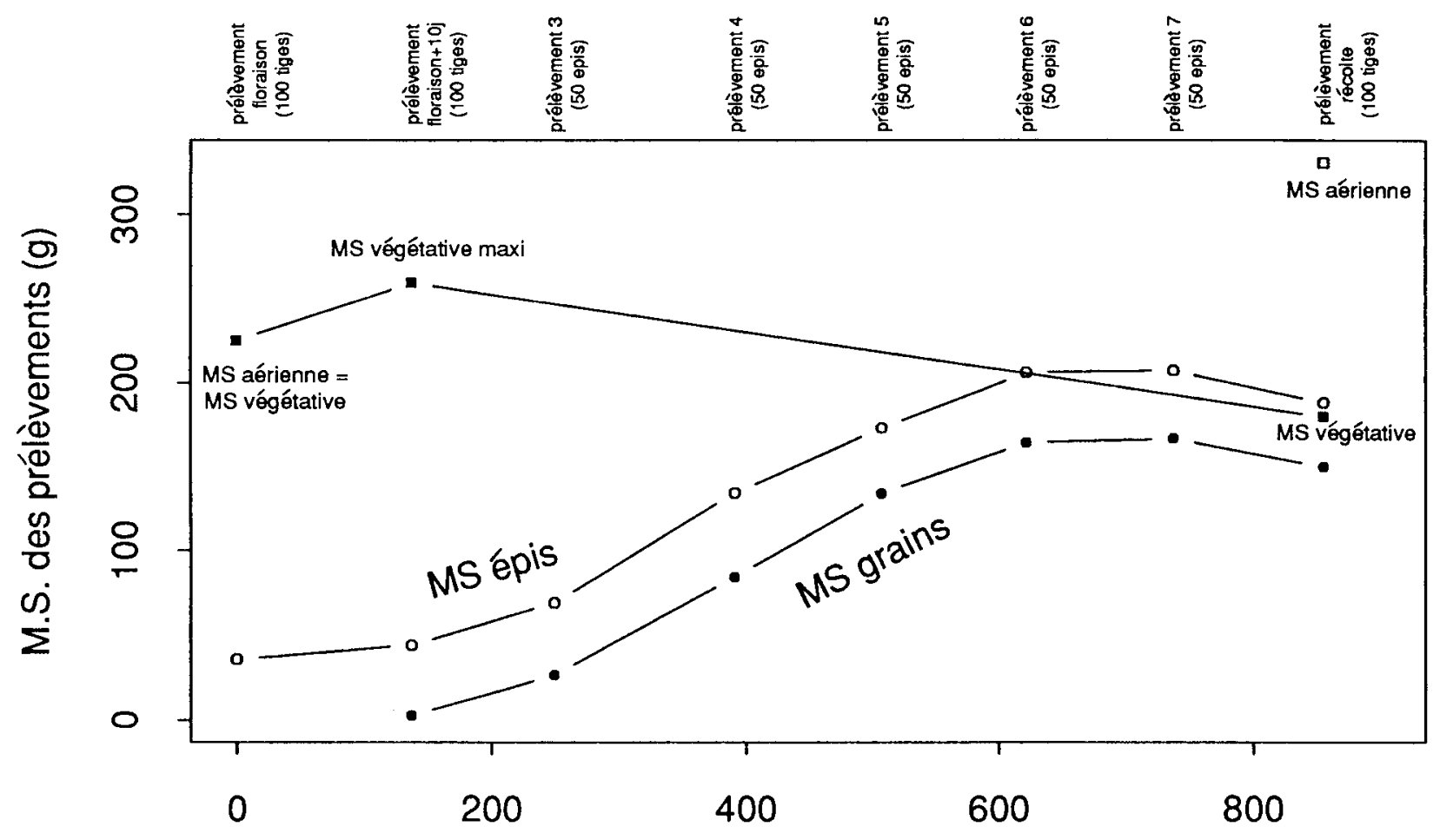

\section{sommes de températures à partir de la floraison (dj)}

Fig 1. Visualisation des différentes valeurs de matière sèche (MS) fournies par l'ensemble des prélèvements (exemple de la variété "Renan», utilisée comme parent mâle, dans le lieu Mons). 
ment réalisé $10 \mathrm{j}$ après la floraison et le prélèvement suivant, ce battage a dû être réalisé avec beaucoup de précaution, compte-tenu des très faibles tailles et poids des grains à ces stades.

Ces poids de 1000 grains ont servi au calcul des vitesses de remplissage. En effet, la croissance de la MS du grain suit classiquement une courbe d'allure sigmoïde, et la vitesse de remplissage d'un grain (v) peut être approchée par la pente de la droite de régression linéaire ajustée sur les points correspondant à la phase d'accumulation rapide de la MS (Sofield et al, 1977; Stoy, 1980; Triboï et al, 1985). On calcule ensuite la durée de remplissage d'un grain (d) à partir de la relation $p m g / 1000=v d$, pmg étant le poids de 1000 grains maximal obtenu lors des 3 derniers prélèvements. À partir de $v$ et $N G$, on obtient la vitesse de croissance des grains par $m^{2}\left(V m^{2}=v N G\right)$, qui correspond au flux d'assimilats vers les grains au niveau du peuplement (Triboï, 1990).

La figure 2 visualise, pour la variété «Renan» à Mons, cette approche de la cinétique de remplissage des grains.

La décomposition du rendement a été faite de plusieurs manières, afin de prendre en compte les différentes relations de compensation entre les composantes (Triboï et Lemercier, 1985) :

$$
\begin{aligned}
R D T & =N G(P M G / 1000) \\
& =N E . N G E . V . d \\
& \quad \operatorname{car} N G=N E . N G E \text { et } P M G / 1000=v . d \\
& =V m^{2} \cdot d \quad \operatorname{car} V m^{2}=N G . V
\end{aligned}
$$

La relation de compensation entre NE et $N G E$, qui détermine $N G$, caractérise la fertilité des génotypes.
Les relations entre $N G$ et $P M G$, ou entre $V m^{2}$ et $d$, qui déterminent le rendement, caractérisent les capacités des génotypes à remplir leurs grains. Les valeurs utilisées pour les différentes représentations graphiques et les ajustements aux modèles sont les moyennes des valeurs obtenues sur les 2 répétitions. II faut noter qu'à Mons les composantes PMG, NGE, NG et NE n'ont pas été mesurées sur les 2 répétitions permettant d'estimer le rendement, mais à partir des résultats des prélèvements.

Les traitements graphiques et statistiques ont été réalisés à l'aide du logiciel «S» (Becker et al, 1988), sur un serveur SUN4-330 sous système d'exploitation UNIX. Pour comparer 2 régressions linéaires, l'une caractéristique des hybrides, l'autre des parents, nous avons utilisé la méthode proposée par Tomassone et al (1983).

\section{RÉSULTATS ET DISCUSSION}

Le niveau moyen des rendements $(88,9 \mathrm{q} / \mathrm{ha}$ à Clermont; 92,3q/ha au Moulon; $88,7 \mathrm{q} / \mathrm{ha}$ à Mons), et les coefficients de variation ( $5 \%$ à Clermont; $7 \%$ au Moulon; $4 \%$ à Mons) indiquent une bonne qualité des 3 essais.

Une analyse de variance effectuée pour la variable $R D T$ met en évidence une forte interaction "génotype-lieu", aussi bien pour les hybrides que pour les parents (tableau I). Par ailleurs, l'étude des corrélations entre lieux pour chacune des variables indique :

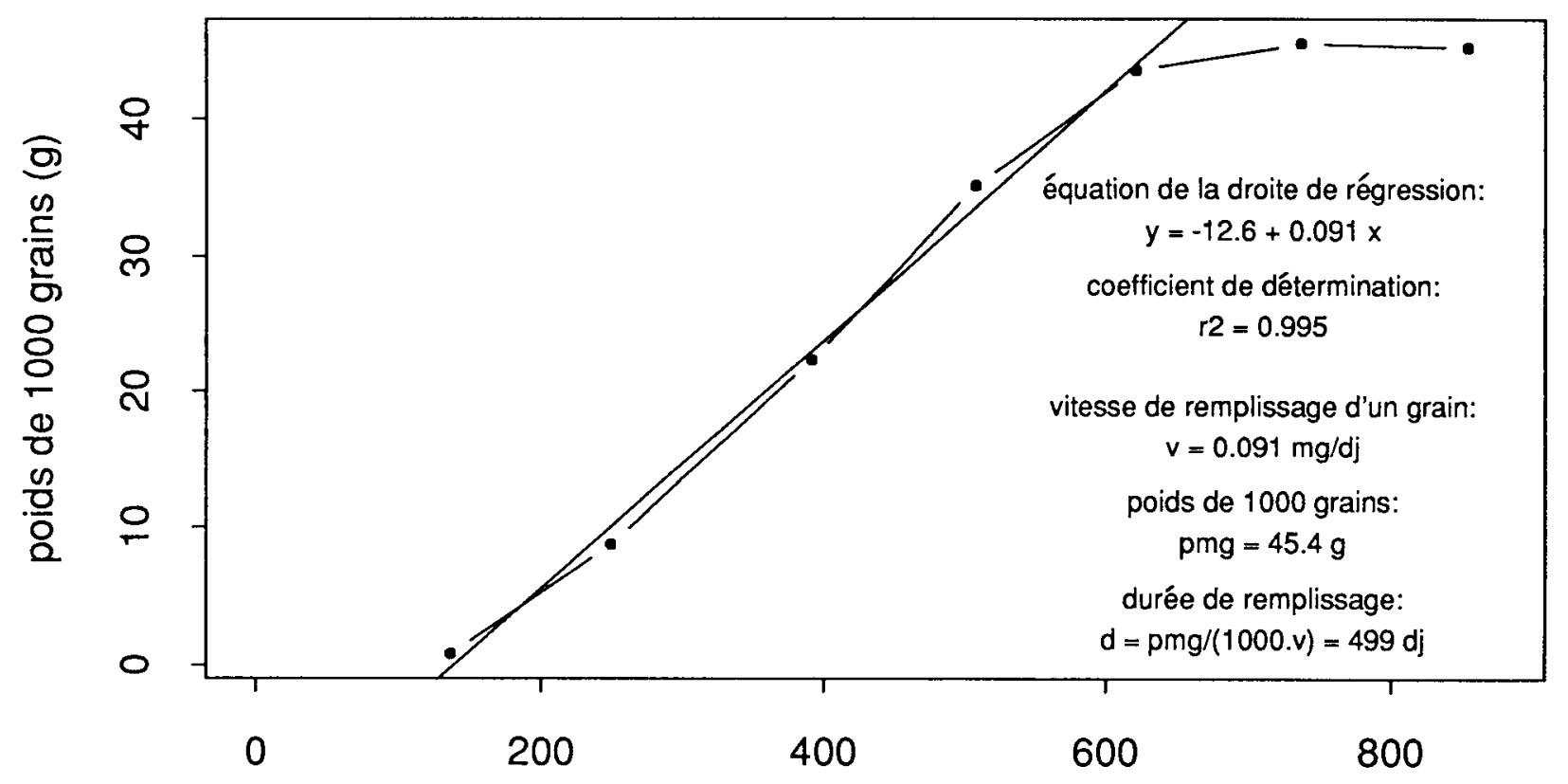

sommes de températures à partir de la floraison (dj)

Fig 2. Estimation des paramètres de vitesse et durée de remplissage (exemple de la variété «Renan" dans le lieu Mons). 
Tableau I. Analyse de variance inter-lieux sur la variable RDT, pour les parents et les hybrides.

\begin{tabular}{|c|c|c|c|c|c|}
\hline \multirow[t]{2}{*}{$R D T$} & \multirow[b]{2}{*}{ Lieu } & \multicolumn{3}{|c|}{ Carrés moyens } & \multirow[b]{2}{*}{ Erreur } \\
\hline & & $\begin{array}{c}\text { Bloc } \\
\text { hiérarchisé à lieu }\end{array}$ & Génotype & $\begin{array}{c}\text { Interaction } \\
\text { génotype-lieu }\end{array}$ & \\
\hline Parents & $\begin{array}{l}161,2^{\star \star \star} \\
(2 \mathrm{ddl})\end{array}$ & $\begin{array}{l}6,4 \mathrm{NS} \\
(3 \mathrm{ddl})\end{array}$ & $\begin{array}{l}129,6^{\star \star \star} \\
(22 \mathrm{ddl})\end{array}$ & $\begin{array}{l}49^{\star \star \star} \\
(42 \mathrm{ddl})\end{array}$ & 16,5 \\
\hline Hybrides & $\begin{array}{l}158,2^{\star \star \star} \\
(2 \mathrm{ddl})\end{array}$ & $\begin{array}{l}15,4 \mathrm{NS} \\
\text { (3 ddl) }\end{array}$ & $\begin{array}{l}92,6^{\star * \star} \\
28 \mathrm{ddl})\end{array}$ & $\begin{array}{l}49,5^{\star \star \star} \\
(55 \mathrm{ddl})\end{array}$ & 19,9 \\
\hline
\end{tabular}

NS $=$ non significatif; ${ }^{* * *}=$ significatif au seuil de $1 \%$.

- que pour un couple de lieux donné ces corrélations sont souvent du même ordre de grandeur pour les hybrides et les lignées, ce qui traduit des comportements similaires ;

- qu'une partie de ces corrélations sont significatives, mais que leur niveau n'est cependant pas suffisant pour que l'on puisse dire que l'information est redondante d'un lieu à l'autre : la valeur moyenne de ces corrélations est de 0,473 , et elles vont de $-0,390$ (pour les parents et la variable ASS, entre Le Moulon et Mons), à +0,861 (pour les parents et la variable $P M G$, entre Clermont et Mons).

Ces différents résultats justifient l'approche de l'élaboration du rendement lieu par lieu.

Le rendement moyen des hybrides a été de $90,9 q /$ ha à Clermont, $95 q /$ ha au Moulon et $92,7 q /$ ha à Mons, pour des rendements moyens respectivement de $86,3 \mathrm{q} / \mathrm{ha}, 88,5 \mathrm{q} / \mathrm{ha}$ et $84,5 \mathrm{q} / \mathrm{ha}$ pour les parents, et $89,2 \mathrm{q} / \mathrm{ha}, 92,7 \mathrm{q} / \mathrm{ha}$ et $87,6 \mathrm{q} /$ ha pour les témoins (Apollo, Récital, Soissons et Thésée).

Cette supériorité des hybrides sur les lignées est illustrée figure 3 , et se retrouve dans le tableau II qui indique l'existence d'un hétérosis par rapport au parent supérieur pour le rendement, significatif dans 2 lieux sur 3 . Ces valeurs d'hétérosis sont comparables à celles obtenues pour des essais réalisés dans des conditions expérimentales proches des nôtres (Borghi et al, 1988; Brears et al, 1988; Morgan et al, 1989; Oury et al, 1990a,b).

\section{Fertilité des hybrides}

Le tableau II et la figure 3 montrent que, pour les composantes $N E, N G E$ et $N G$, l'hybride se situe au niveau, ou légèrement en dessous, du parent moyen.

La figure 3 montre que la relation de compensation entre NGE et NE peut apparaître lorsqu'on étudie globalement un même lot de génotypes dans différents lieux. C'est ainsi qu'à Mons les peuplements épis obtenus ont été plus faibles qu'à Clermont et au Moulon, mais ont été compensés par des nombres de grains par épi supérieurs.

Par ailleurs, l'étude de la relation entre $N G E$ et $N E$ montre que l'équation de la droite de régression qui caractérise les hybrides n'est pas significativement différente de celle qui caractérise les lignées $\left(F_{2,47}=0,75\right.$ à Clermont : $P>$ 0,$05 ; F_{2,45}=1,36$ au Moulon : $P>0,05 ; F_{2,46}=$ $0,14$ à Mons : $P>0,05)$. De plus, cette relation est assez faible: les coefficients de détermination obtenus sur l'ensemble des génotypes sont de 0,21 à Clermont, 0,43 au Moulon et 0,19 à Mons.

Le fait que dans les 3 lieux on ne révèle pas d'hétérosis pour $N E, N G E$ et $N G$, et que la relation de compensation entre NE et NGE soit équivalente pour les $F_{1}$ et les lignées, indique que les hybrides ne se distinguent de leurs parents ni pour la fertilité (NG), ni pour l'élaboration de ce nombre de grains par $\mathrm{m}^{2}$.

\section{Capacités de remplissage des hybrides}

Le tableau II et la figure 3 indiquent qu'en moyenne il existe dans les 3 lieux un hétérosis par rapport au parent supérieur pour le poids de 1000 grains (cet hétérosis est significatif dans 2 lieux sur 3), et que pour $v$ et $V m^{2}$ l'hybride se 


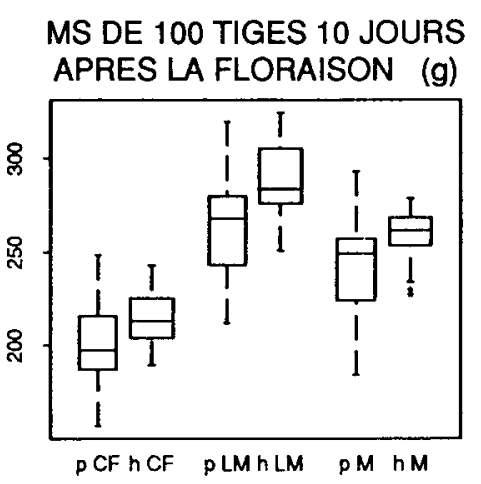

NOMBRE D'EPIS/m2

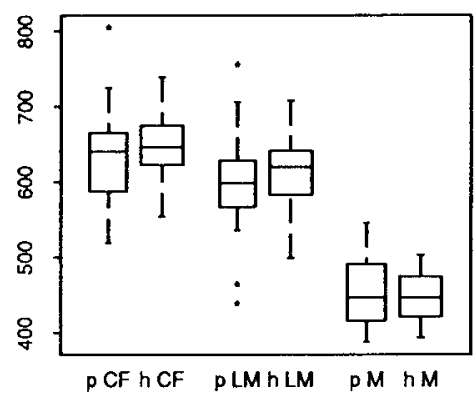

POIDS DE 1000 GRAINS

(g)

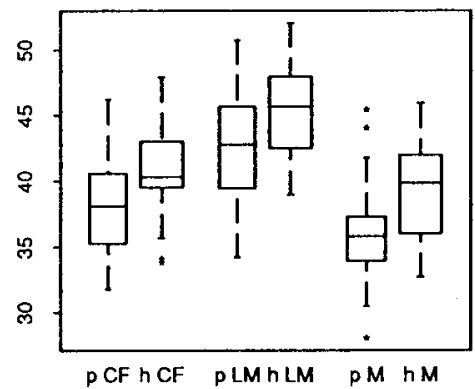

VITESSE DE REMPLISSAGE/m2 (g/dj.m2)

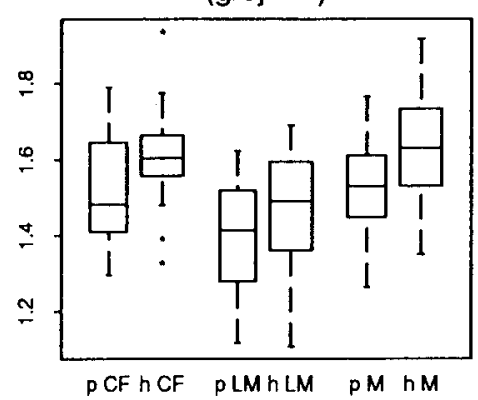

MS AERIENNE A LA RECOLTE (q/ha)

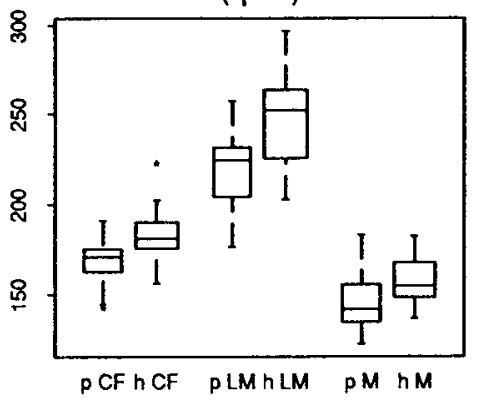

NOMBRE DE GRAINS/EPI

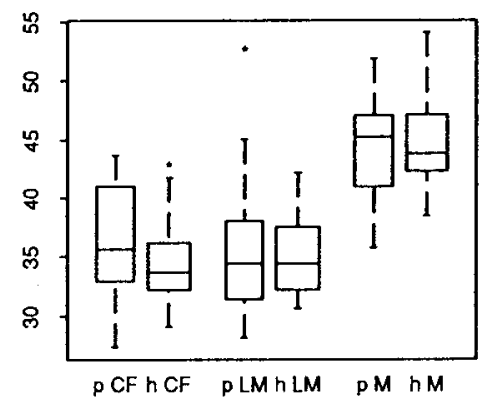

VITESSE DE REMPLISSAGE $(\mathrm{mg} / \mathrm{dj})$

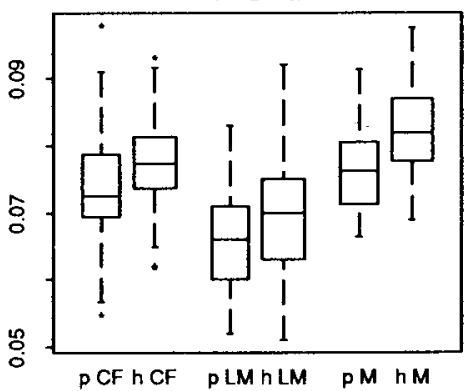

(dj)

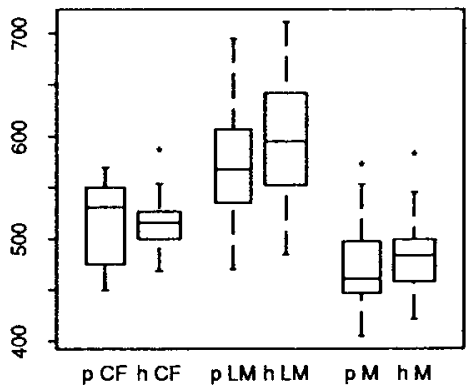

DUREE DE REMPLISSAGE

(dj)

RENDEMENT

(q/ha)

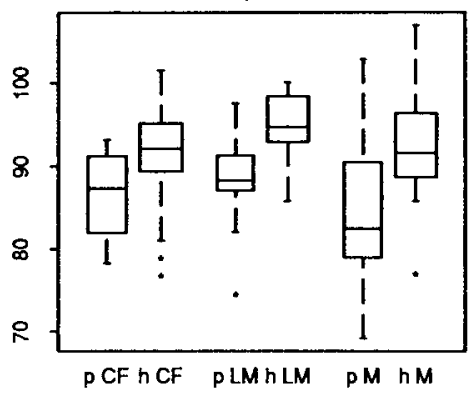

NOMBRE DE GRAINS/m2

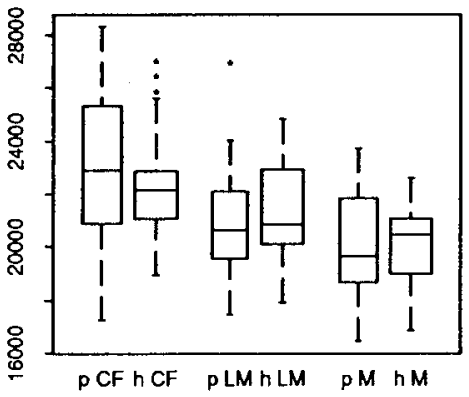

pCF hCF pLMhLM pM hM

Fig 3. Box-plots des différentes variables disponibles dans les 3 lieux pour les parents et les hybrides. CF = Clermont; LM = Le Moulon; $M=$ Mons; $p=$ parents; $h=$ hybrides.
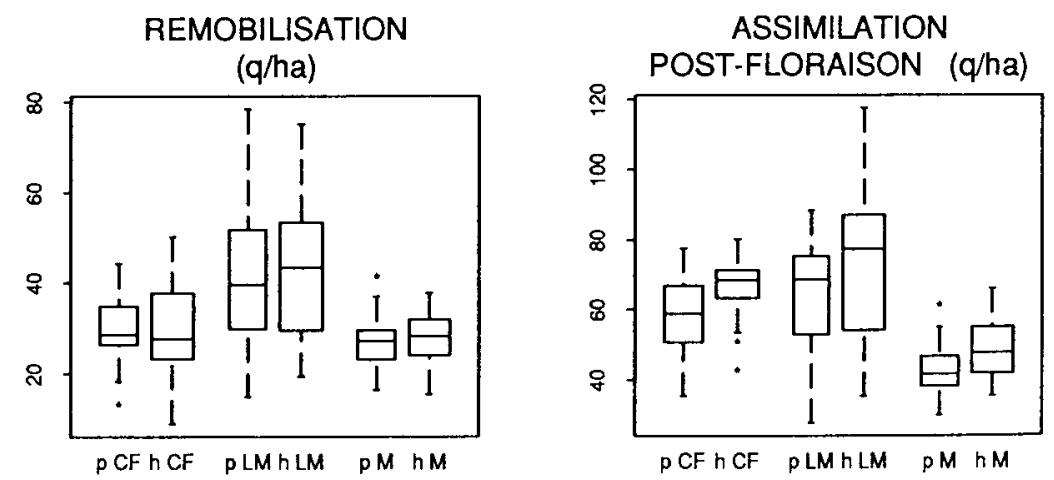
Tableau II. Valeurs d'hétérosis (exprimées en \%) pour les différentes variables étudiées, et résultats du test de Student unilatéral (probabilité associée à la valeur de la statistique, et significativité) permettant de tester la supériorité des hybrides par rapport aux parents moyen et supérieur. Pour chaque variable, la première ligne concerne l'hétérosis par rapport au parent moyen, et la seconde l'hétérosis par rapport au parent supérieur.

\begin{tabular}{|c|c|c|c|c|c|c|c|c|c|}
\hline \multirow[b]{3}{*}{ MSt } & \multicolumn{3}{|c|}{ Clermont } & \multicolumn{3}{|c|}{ Le Moulon } & \multicolumn{3}{|c|}{ Mons } \\
\hline & \multirow{2}{*}{$\begin{array}{l}\text { Hétérosis } \\
+5 \\
+0,4\end{array}$} & \multicolumn{2}{|c|}{ Test $\mathrm{t}$} & \multirow{2}{*}{$\begin{array}{c}\text { Hétérosis } \\
\begin{array}{c}+7,6 \\
+0,2\end{array}\end{array}$} & \multicolumn{2}{|c|}{ Test $\mathrm{t}$} & \multirow{2}{*}{$\begin{array}{c}\text { Hétérosis } \\
\begin{array}{c}+5,8 \\
+0,1\end{array}\end{array}$} & \multicolumn{2}{|c|}{ Test $\mathrm{t}$} \\
\hline & & $\begin{array}{l}0,004 \\
0,628\end{array}$ & NS & & $\begin{array}{l}0,001 \\
0,643\end{array}$ & NS & & $\begin{array}{l}0,001 \\
0,606\end{array}$ & NS \\
\hline MSTaf & $\begin{array}{r}12,5 \\
+5,2\end{array}$ & $\begin{array}{l}0 \\
0\end{array}$ & $\stackrel{\star \star \star *}{* \star \star}$ & $\begin{array}{r}+12,4 \\
+4,5\end{array}$ & $\begin{array}{l}0 \\
0,008\end{array}$ & $\begin{array}{l}\star \star \star * \\
\star \star\end{array}$ & $\begin{array}{r}+11,2 \\
+2,7\end{array}$ & $\begin{array}{l}0 \\
0,016\end{array}$ & 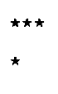 \\
\hline$M S r$ & $\begin{array}{l}+8,4 \\
+5,2\end{array}$ & $\begin{array}{l}0 \\
0\end{array}$ & $\begin{array}{l}\star \star \star \star \\
\star \star \star\end{array}$ & $\begin{array}{r}+10,5 \\
+6,2\end{array}$ & $\begin{array}{l}0 \\
0,005\end{array}$ & $\begin{array}{l}\star \star \star \\
\star \star\end{array}$ & $\begin{array}{l}+8,3 \\
+2,8\end{array}$ & $\begin{array}{l}0 \\
0,069\end{array}$ & $\begin{array}{l}\star \star \star \\
\left({ }^{*}\right)\end{array}$ \\
\hline$R D T$ & $\begin{array}{l}+5,5 \\
+3\end{array}$ & $\begin{array}{l}0 \\
0,005\end{array}$ & $\stackrel{\star \star \star}{\star \star}$ & $\begin{array}{l}+5,2 \\
+2,4\end{array}$ & $\begin{array}{l}0,001 \\
0,123\end{array}$ & $\begin{array}{l}\star \star \star \\
\text { NS }\end{array}$ & $\begin{array}{l}+9 \\
+4\end{array}$ & $\begin{array}{l}0 \\
0,001\end{array}$ & 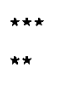 \\
\hline$I R$ & $\begin{array}{l}-1,5 \\
-4,4\end{array}$ & $\begin{array}{l}0,976 \\
0,999\end{array}$ & $\begin{array}{l}\text { NS } \\
\text { NS }\end{array}$ & $\begin{array}{l}-1,3 \\
-4,5\end{array}$ & $\begin{array}{l}0,869 \\
0,999\end{array}$ & $\begin{array}{l}\text { NS } \\
\text { NS }\end{array}$ & $\begin{array}{l}+1,5 \\
-1,3\end{array}$ & $\begin{array}{l}0,051 \\
0,918\end{array}$ & $\begin{array}{l}\left({ }^{\star}\right) \\
\text { NS }\end{array}$ \\
\hline$N E$ & $\begin{array}{l}-2,5 \\
-7,6\end{array}$ & $\begin{array}{l}0,962 \\
0,999\end{array}$ & $\begin{array}{l}\text { NS } \\
\text { NS }\end{array}$ & $\begin{array}{l}-0,1 \\
-5,3\end{array}$ & $\begin{array}{l}0,612 \\
0,988\end{array}$ & $\begin{array}{l}\text { NS } \\
\text { NS }\end{array}$ & $\begin{array}{l}-3,2 \\
-7,9\end{array}$ & $\begin{array}{l}0,993 \\
0,999\end{array}$ & $\begin{array}{l}\text { NS } \\
\text { NS }\end{array}$ \\
\hline NGE & $\begin{array}{l}-0,3 \\
-6,4\end{array}$ & $\begin{array}{l}0,68 \\
0,999\end{array}$ & $\begin{array}{l}\text { NS } \\
\text { NS }\end{array}$ & $\begin{array}{l}-1,7 \\
-8,7\end{array}$ & $\begin{array}{l}0,843 \\
0,999\end{array}$ & $\begin{array}{l}\text { NS } \\
\text { NS }\end{array}$ & $\begin{array}{l}+2,2 \\
-4,1\end{array}$ & $\begin{array}{l}0,043 \\
0,998\end{array}$ & NS \\
\hline$N G$ & $\begin{array}{l}+0,9 \\
-5,1\end{array}$ & $\begin{array}{l}0,997 \\
1\end{array}$ & $\begin{array}{l}\text { NS } \\
\text { NS }\end{array}$ & $\begin{array}{l}-2,3 \\
-7,7\end{array}$ & $\begin{array}{l}0,972 \\
1\end{array}$ & $\begin{array}{l}\text { NS } \\
\text { NS }\end{array}$ & $\begin{array}{l}-1 \\
-6,4\end{array}$ & $\begin{array}{l}0,846 \\
0,999\end{array}$ & $\begin{array}{l}\text { NS } \\
\text { NS }\end{array}$ \\
\hline$P M G$ & $\begin{array}{l}+8,4 \\
+2,3\end{array}$ & $\begin{array}{l}0 \\
0,044\end{array}$ & $\begin{array}{l}\star \star \star \\
\star\end{array}$ & $\begin{array}{r}+7,6 \\
+1,4\end{array}$ & $\begin{array}{l}0 \\
0,189\end{array}$ & NS & $\begin{array}{l}+9,9 \\
+3,4\end{array}$ & $\begin{array}{l}0 \\
0,011\end{array}$ & 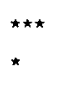 \\
\hline RESaf & $\begin{array}{r}12,9 \\
+2,5\end{array}$ & $\begin{array}{l}0 \\
0,344\end{array}$ & NS & $\begin{array}{r}+15,9 \\
+7,3\end{array}$ & $\begin{array}{l}0 \\
0,004\end{array}$ & $\begin{array}{l}* \star \star \\
* \star\end{array}$ & $\begin{array}{l}+8,4 \\
-1,4\end{array}$ & $\begin{array}{l}0 \\
0,887\end{array}$ & NS \\
\hline$v$ & $\begin{array}{l}+5,7 \\
-2,4\end{array}$ & $\begin{array}{l}0 \\
0,966\end{array}$ & NS & $\begin{array}{l}+7,3 \\
+0,1\end{array}$ & $\begin{array}{l}0,004 \\
0,606\end{array}$ & $\stackrel{\text { NS }}{\text { N* }}$ & $\begin{array}{l}+6,9 \\
+2,2\end{array}$ & $\begin{array}{l}0 \\
0,089\end{array}$ & $\begin{array}{l}* \star * \\
(*)\end{array}$ \\
\hline$d$ & $\begin{array}{l}-0,1 \\
-4,2\end{array}$ & $\begin{array}{l}0,626 \\
0,998\end{array}$ & $\begin{array}{l}\text { NS } \\
\text { NS }\end{array}$ & $\begin{array}{l}+2,3 \\
-2,4\end{array}$ & $\begin{array}{l}0,206 \\
0,876\end{array}$ & $\begin{array}{l}\text { NS } \\
\text { NS }\end{array}$ & $\begin{array}{l}+3 \\
-0,5\end{array}$ & $\begin{array}{l}0,027 \\
0,692\end{array}$ & NS \\
\hline$V m 2$ & $\begin{array}{l}+7,2 \\
+2,4\end{array}$ & $\begin{array}{l}0 \\
0,1\end{array}$ & $\begin{array}{l}* \star * \\
\left({ }^{\star}\right)\end{array}$ & $\begin{array}{l}+5,3 \\
-0,2\end{array}$ & $\begin{array}{l}0,036 \\
0,593\end{array}$ & NS & $\begin{array}{l}+6,3 \\
+2,1\end{array}$ & $\begin{array}{l}0,002 \\
0,160\end{array}$ & NS \\
\hline REM & $\begin{array}{r}-1,5 \\
-11,7\end{array}$ & $\begin{array}{l}0,653 \\
0,976\end{array}$ & $\begin{array}{l}\text { NS } \\
\text { NS }\end{array}$ & $\begin{array}{r}+10,4 \\
-2,4\end{array}$ & $\begin{array}{l}0,354 \\
0,993\end{array}$ & $\begin{array}{l}\text { NS } \\
\text { NS }\end{array}$ & $\begin{array}{l}+9,1 \\
-6,2\end{array}$ & $\begin{array}{l}0,087 \\
0,974\end{array}$ & $\begin{array}{l}\left({ }^{\star}\right) \\
\text { NS }\end{array}$ \\
\hline ASS & $\begin{array}{l}+16 \\
+6,2\end{array}$ & $\begin{array}{l}0 \\
0,068\end{array}$ & $\begin{array}{l}\star \star * \\
\left({ }^{\star}\right)\end{array}$ & $\begin{array}{l}+21 \\
+1,4\end{array}$ & $\begin{array}{l}0,005 \\
0,502\end{array}$ & ${ }^{\star \star}$ NS & $\begin{array}{c}+15,5 \\
+7\end{array}$ & $\begin{array}{l}0,001 \\
0,100\end{array}$ & $\begin{array}{l}\star \star \\
\left({ }^{\star}\right)\end{array}$ \\
\hline
\end{tabular}

$M S f=M S$ aérienne à la floraison; $M S T a f=M S$ de la tige $10 \mathrm{j}$ après la floraison; $M S r=M S$ aérienne à la récolte; $R D T=$ rendement; $I R=$ indice de récolte; $N E=$ nombre d'épis par $\mathrm{m}^{2} ; N G E=$ nombre de grains par épi; $N G=$ nombre de grains par $\mathrm{m}^{2} ; P M G=$ poids de 1000 grains; RESaf $=$ MSTaf/NGE = indicateur de la quantité d'assimilats potentiellement disponibles pour le remplissage du grain; $v=$ vitesse de remplissage; $d=$ durée de remplissage; $V m^{2}=$ vitesse de remplissage par $m^{2} ; R E M=$ remobilisation; $A S S=$ assimilation post-floraison. NS = non significatif; (") = significatif au seuil de $10 \% ;{ }^{*}=$ significatif au seuil de $5 \%$; ${ }^{* *}=$ significatif au seuil de $1 \% ;{ }^{* \star \star}=$ significatif au seuil de $1 \%$. 
situe au niveau du parent supérieur. A propos de $v$, on peut noter que l'ajustement permettant d'obtenir la vitesse de remplissage (fig 2) est généralement de très bonne qualité : les valeurs moyennes des coefficients de détermination ont été de 0,995 à Clermont, 0,991 au Moulon et 0,996 à Mons.

Pour la durée de remplissage, l'hybride est proche du parent moyen à Clermont, entre parent moyen et parent supérieur au Moulon, et proche du parent supérieur à Mons.

La figure 4 montre que la relation de compensation entre PMG et NG est forte (les coefficients de détermination sont de l'ordre de $60 \%$ ), et qu'on obtient une meilleure analyse de la relation lorsqu'on distingue la sous-population des hybrides de celle des parents $\left(F_{2,47}=7,24\right.$ a Clermont: $P<0,01 ; F_{2,45}=12,6$ au Moulon : $P<0,01 ; F_{2,46}=9,85$ à Mons: $\left.P<0,01\right)$.

Dans les 3 lieux, la relation de compensation entre NG et $P M G$ ne se fait donc pas au même niveau pour les lignées et pour les $F_{1}$ : les hybrides se distinguent des lignées par des capacités de remplissage supérieures. Le fait qu'on ait un hétérosis pour $P M G$ et pas d'hétérosis pour $N G$ indique que cette différence dans les capacités de remplissage correspond généralement à une $F_{1}$ qui, pour un nombre de grains par $\mathrm{m}^{2}$ équivalent à celui de ses parents, obtient un poids de 1000 grains supérieur.

La relation de compensation entre $V m^{2}$ et $d$ qui, comme la relation entre $N G$ et $P M G$, détermine le rendement des génotypes, permet de retrouver d'une autre manière la supériorité des hybrides pour les capacités de remplissage. La figure 5 montre que la relation entre $V m^{2}$ et $d$ est assez forte (sauf dans le cas des parents à Mons, pour lequel le coefficient de détermination vaut seulement 0,17 ), et que la compensation ne se fait pas au même niveau pour les parents et pour les hybrides $\left(\mathrm{F}_{2,47}=4,33\right.$ à Clermont : $P<$ 0,$05 ; \mathrm{F}^{2,45}=5,52$ au Moulon: $P<0,01 ; \mathrm{F}_{2,46}=$ 4,36 à Mons : $P<0,05$ ).

Cependant, la différence dans les capacités de remplissage mise ainsi en évidence peut correspondre:

- soit à une $F_{1}$ présentant une vitesse de croissance des grains au $\mathrm{m}^{2}$ supérieure à celle de ses parents, tout en ayant une durée de remplissage équivalente;

- soit à une $F_{1}$ bénéficiant d'une durée de remplissage supérieure, pour une vitesse de remplissage par $\mathrm{m}^{2}$ équivalente à celle de ses parents.
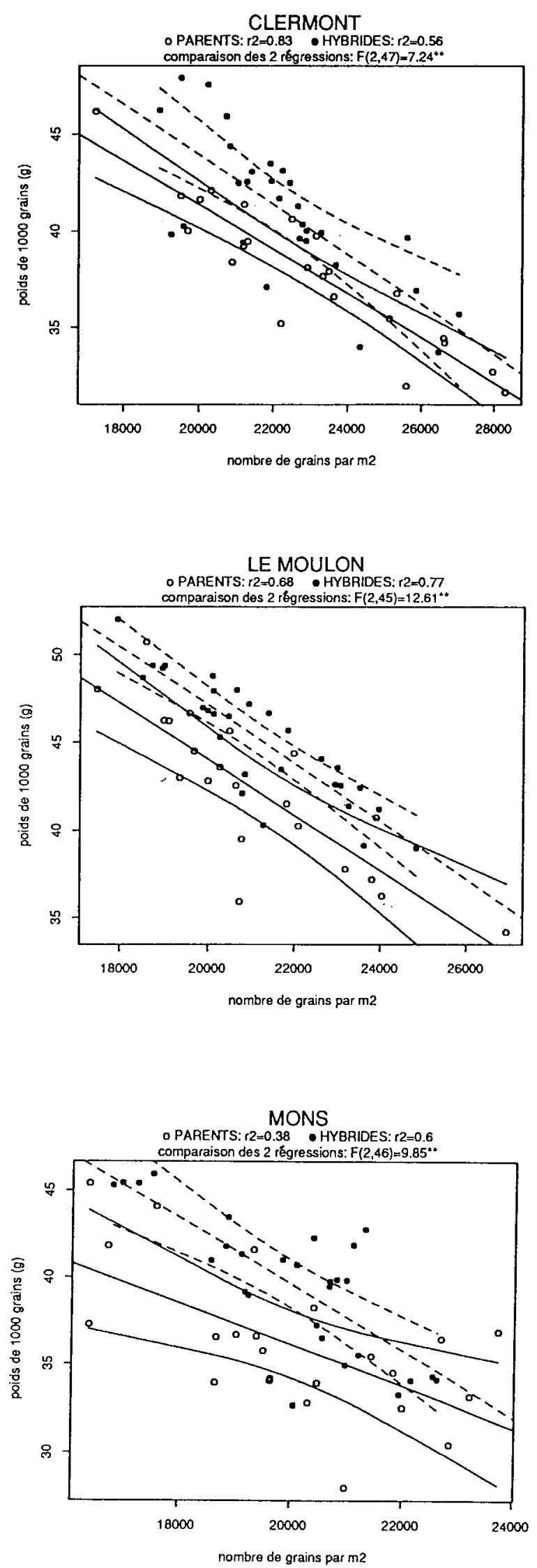

Fig 4. Relations de compensation entre NG et $P M G$ dans les 3 lieux (la droite de régression caractérisant les hybrides est en pointillés; les courbes encadrant les droites de régressions délimitent la région de confiance à $95 \%$ de ces droites). 

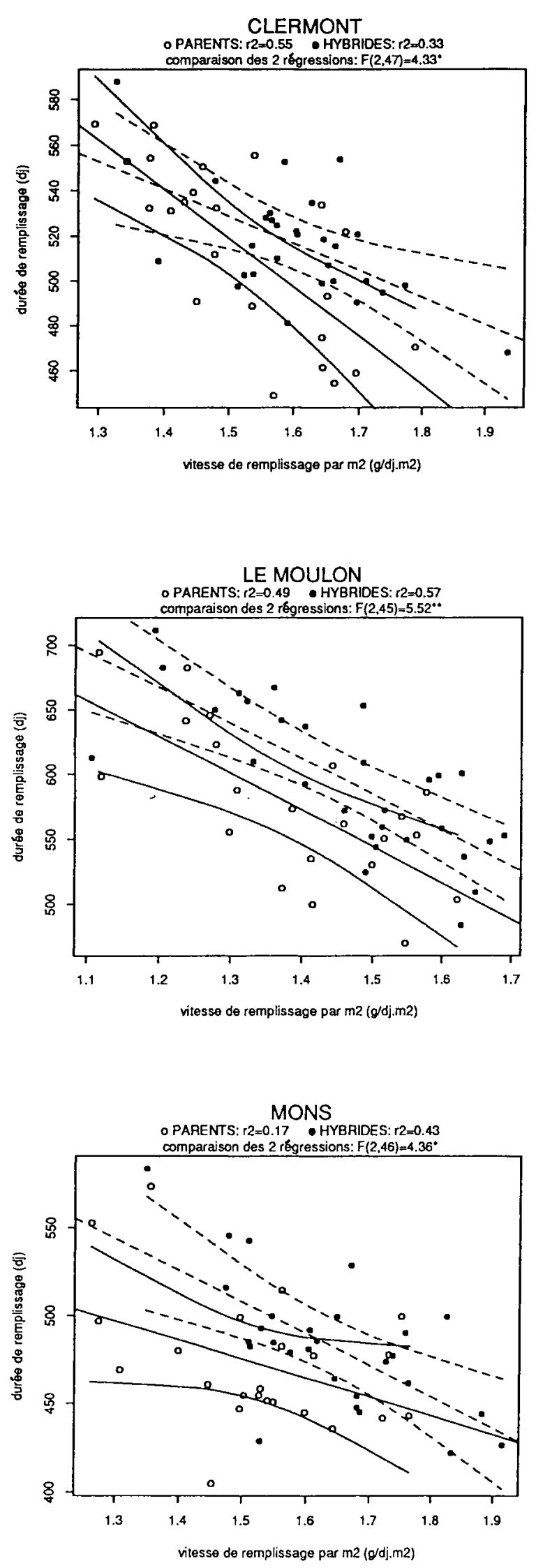

Fig 5. Relations de compensation entre $\mathrm{Vm}^{2}$ et $d$ dans les 3 lieux (la droite de régression caractérisant les hybrides est en pointillés; les courbes encadrant les droites de régressions délimitent la région de confiance à $95 \%$ de ces droites).
En effet, bien qu'on ait des valeurs d'hétérosis plus grandes pour $V m^{2}$ que pour $d$, il faut être plus prudent ici que dans le cas des variables $N G$ et $P M G$, et cela pour 3 raisons:

- parce que les valeurs d'hétérosis de $V m^{2}$ et $d$ sont moins contrastées que celles de $P M G$ et $N G$;

- parce que la compensation entre $V m^{2}$ et $d$ est moins forte (les coefficients de détermination sont de l'ordre de $40 \%$, alors qu'ils étaient de l'ordre de $60 \%$ pour la compensation entre PMG et $N G$ ) ;

- parce que la relation caractérisant les hybrides n'est différente de celle caractérisant les parents qu'au seuil de $5 \%$ (pour la compensation entre $P M G$ et NG, la différence entre hybrides et parents était significative au seuil de $1 \%$ dans les 3 lieux).

En fait, la relation entre $V m^{2}$ et $d$ peut être décomposée, d'abord en une relation entre $N G$ et $v$, et ensuite en une relation entre $v$ et $d$.

En ce qui concerne la relation entre $N G$ et $v$, la figure 6 montre qu'il y a une compensation assez forte entre ces 2 composantes (les coefficients de détermination sont au moins de l'ordre de $30 \%$ ), mais la régression caractérisant les $\mathrm{F} 1$ ne diffère significativement de la régression caractérisant les parents que dans le cas de Clermont et de Mons $\left(\mathrm{F}_{2,47}=3,8\right.$ à Clermont : $P<$ 0,$05 ; F_{2,45}=1,44$ au Moulon : $P>0,05 ; F_{2,46}=$ 5,29 à Mons : $P<0,01$ ).

Pour la relation entre $v$ et $d$, on peut d'abord remarquer (fig 3) qu'elle apparaît lorsqu'on considère globalement les génotypes étudiés, dans les 3 lieux : c'est ainsi qu'au Moulon les vitesses de remplissage ont été en moyenne plus faibles qu'à Clermont et Mons, mais ont été compensées par des durées de remplissage plus élevées. En faisant l'analyse lieu par lieu, on obtient des résultats différents pour les 3 localisations (fig 6) :

- à Clermont, il y a une compensation forte entre $v$ et $d$ dans le cas des parents $\left(r^{2}=0,54\right)$, et pas de compensation dans le cas des hybrides $\left(r^{2}=0,07\right)$;

- au Moulon, la compensation entre $v$ et $d$ est assez forte (les coefficients de détermination sont de l'ordre de $40 \%$ ), et elle ne se fait pas au même niveau pour les lignées et pour les hybrides ;

- à Mons, il n'apparaît pas de compensation entre $v$ et $d$, ni pour les lignées $\left(r^{2}=0,01\right)$, ni pour les hybrides $\left(r^{2}=0,09\right)$. 

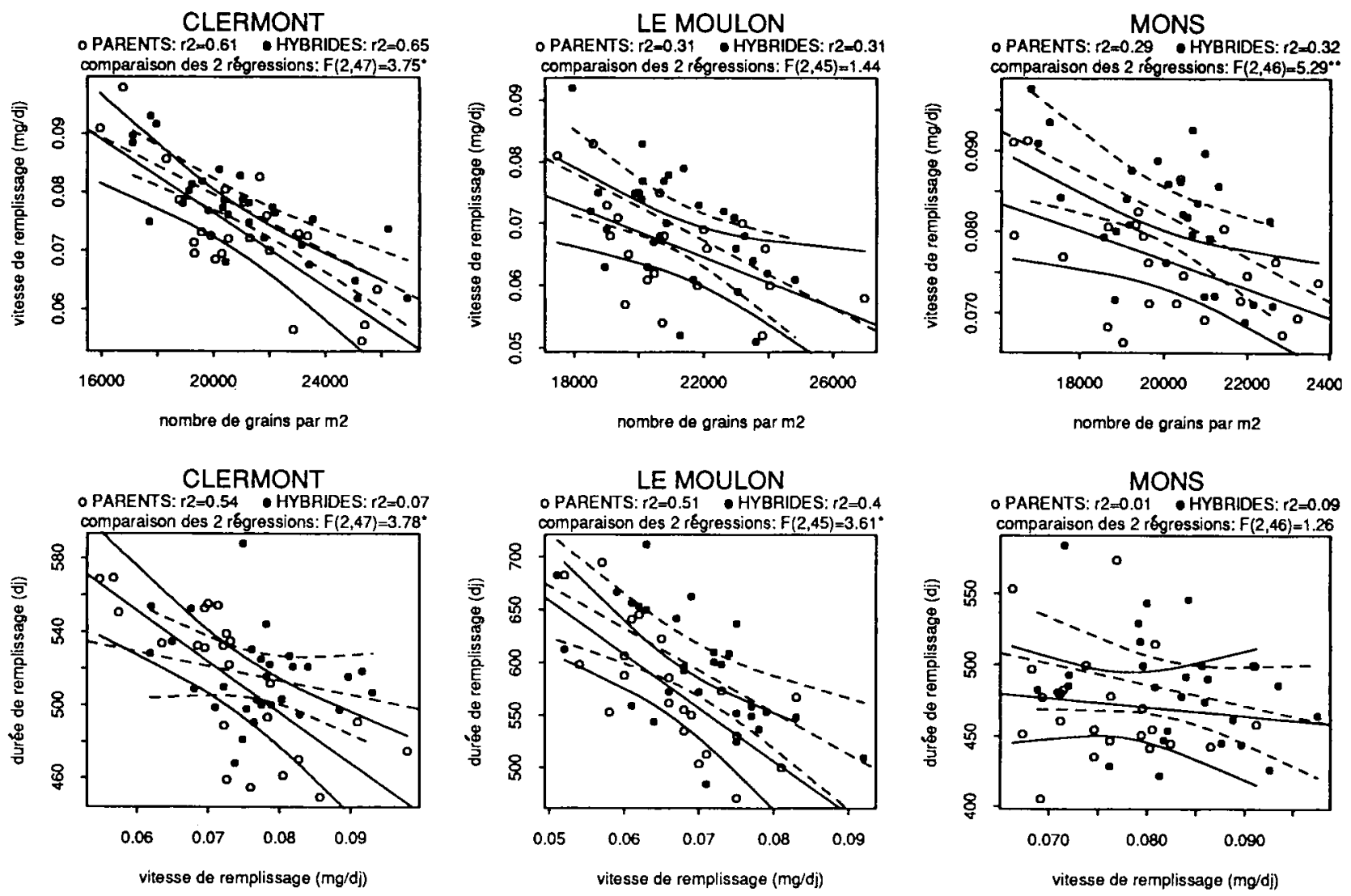

Fig 6. Relations de compensation entre NG et $v$ d'une part, et $v$ et $d$ d'autre part, dans les 3 lieux (la droite de régression caractérisant les hybrides est en pointillés; les courbes encadrant les droites de régressions délimitent la région de confiance à $95 \%$ de ces droites).

Ces résultats concernant les relations entre $N G$ et $v$, et entre $v$ et $d$, peuvent être interprétés de la manière suivante:

- au Moulon, l'hétérosis observé pour $v$ apparaît insuffisant pour que la compensation entre $v$ et NG se fasse à un niveau significativement différent chez les hybrides et chez les lignées. Cette différence avec les 2 autres lieux vient du fait qu'au Moulon les vitesses de remplissage ont été en moyenne inférieures: I'hétérosis pour la vitesse de remplissage, bien que prenant une valeur similaire à celle obtenue à Mons, correspond alors à un plus faible écart entre hybrides et lignées. On peut alors penser que l'hétérosis observé pour $V m^{2}$ n'est pas non plus suffisant pour expliquer la différence significative entre hybrides et parents au niveau de la compensation entre $V m^{2}$ et $d$. Au Moulon, la supériorité de l'hybride pour les capacités de remplissage serait donc due à un comportement intermédiaire entre celui d'une $F_{1}$ qui aurait une vitesse de remplissage par $\mathrm{m}^{2}$ supérieure à celle de ses parents, tout en ayant une durée de remplissage équiva- lente, et celui d'une $F_{1}$ qui bénéficierait d'une durée de remplissage supérieure à celle de ses parents, pour une vitesse de remplissage par $\mathrm{m}^{2}$ équivalente. Cette interprétation est tout à fait compatible avec ce qu'on observe pour la relation entre $v$ et $d$. En effet, au Moulon la compensation entre vitesse et durée de remplissage se fait à un niveau différent pour les hybrides et les lignées. Comme les valeurs d'hétérosis ne sont pas très contrastées pour $v$ et $d$, on peut penser que la différence entre hybrides et lignées correspond effectivement à une supériorité de l'hybride sur ses parents à la fois pour la vitesse et la durée (la vitesse étant cependant prédominante);

- à Clermont et à Mons, l'hétérosis obtenu pour $v$ est suffisant pour que la compensation entre $N G$ et $v$ se fasse à des niveaux significativement différents chez les $F_{1}$ et chez les lignées. Dans ces 2 lieux, l'hybride obtient donc généralement une vitesse de remplissage plus élevée pour un nombre de grains par $\mathrm{m}^{2}$ équivalent à celui de ses parents, et ceci est suffisant pour expliquer 
la supériorité de l'hybride pour les capacités de remplissage. Cependant, une durée de remplissage plus importante chez l'hybride que chez ses parents est également possible à Mons car, dans ce lieu, l'hétérosis observé pour $d$ n'est pas très inférieur à celui observé pour $v$.

En résumé, on peut dire que les plus grandes capacités de remplissage de l'hybride sont dues essentiellement à une supériorité de la $F_{1}$ sur ses parents pour la vitesse de remplissage, qui peut être en plus renforcée par une supériorité au niveau de la durée de remplissage (ce qui est le cas à Mons, et surtout au Moulon).

\section{Origine de la supériorité des hybrides pour la vitesse de remplissage}

Le tableau II montre que, pour la production de MS aérienne par $\mathrm{m}^{2}$ à la floraison, l'hybride se situe au niveau du parent supérieur. Dix jours après la floraison, on constate qu'il y a un hétérosis pour le poids d'une tige (tableau II et fig 3), et que celui-ci est significatif dans les 3 lieux. Cet hétérosis pour le poids d'une tige pourrait être en grande partie expliqué par une augmentation de la taille des tiges chez la $F_{1}$. Au Moulon, où la hauteur a été mesurée, on observe en effet un hétérosis de $+4 \%$ par rapport au parent supérieur pour cette variable, et une forte corrélation positive entre hauteur et poids d'une tige (pour cette relation, il n'y a pas de différence significative entre parents et hybrides: $F_{2,45}=0,82, P>0,05$; et le coefficient de détermination calculé sur l'ensemble des génotypes est de 0,66).

$\mathrm{Si}$ on considère que la MS d'une tige $10 \mathrm{j}$ après la floraison est directement proportionnelle, à travers le stock de réserves qu'elle représente, à la quantité d'assimilats potentiellement utilisables pour le remplissage des grains, on peut en conclure que l'hétérosis observé correspond à des disponibilités en assimilats plus importantes chez l'hybride que chez ses parents. En rapportant le poids d'une tige au nombre de grains portés par cette tige, on obtient alors un indicateur (RESaf) de la quantité d'assimilats potentiellement utilisable pour le remplissage d'un grain. Nos données font apparaître une forte corrélation positive entre cet indicateur et la vitesse de remplissage: il n'y a pas de différence significative entre hybrides et parents pour cette relation $\left(\mathrm{F}_{2,47}=0,29\right.$ à Clermont : $P>0,05 ; \mathrm{F}_{2,45}=0,28$ au Moulon : $P>0,05 ; \mathrm{F}_{2,46}=3,1$ à Mons : $P>$ $0,05)$, et les coefficients de détermination obte- nus sur l'ensemble des génotypes sont de 0,50 à Clermont, 0,25 au Moulon et 0,49 à Mons. Par ailleurs, le tableau II montre que la $F_{1}$ est au moins au niveau du parent supérieur pour cette variable RESaf.

À la floraison, on peut considérer que la relation «source-puits» est installée. En effet, le poids des racines et l'indice foliaire atteignent leur maximum approximativement au moment de l'épiaison (Gallagher et al, 1975; Biscoe et al, 1975) : les potentiels d'absorption racinaire et photosynthétique (la source) sont donc fixés. De même, le nombre d'épis et le nombre de grains par épi sont fixés à la fin de la montaison (Masle-Meynard, 1980), et le potentiel reproductif correspondant au nombre de grains (les puits) est donc également fixé à la floraison. Nos résultats indiquent que la situation hybride aurait pour effet d'augmenter la quantité d'assimilats disponibles par grain au début de la période de remplissage, et induirait ainsi une relation "sourcepuits" différente chez la $F_{1}$, notamment en augmentant le flux d'assimilats vers le grain.

\section{Maintien d'une relation "source-puits" plus favorable chez l'hybride}

Le tableau II et la figure 3 montrent qu'il existe un hétérosis pour la production de MS aérienne à la récolte. Le fait que cet hétérosis soit associé à un hétérosis pour le rendement en grains conduit à un indice de récolte chez l'hybride sensiblement équivalent à celui du parent moyen (tableau II). La voie hybride apparaîtrait alors comme une manière d'augmenter le rendement à travers une augmentation de la production de MS. En cela, elle pourrait constituer une voie d'amélioration du blé originale. En effet, d'après les résultats de Austin et al (1980), les progrès enregistrés dans la sélection des lignées auraient, eux, été réalisés pour l'essentiel à production de MS constante, par une augmentation de l'indice de récolte.

L'hétérosis pour la production de MS apparaît plus marqué à la récolte qu'à la floraison (pour $M S r$, l'hétérosis par rapport au parent supérieur est significatif dans les 3 lieux). Ce renforcement de l'hétérosis peut être relié à ce qu'on observe pour l'assimilation post-floraison: il y a un hétérosis par rapport au parent supérieur pour ASS, et cet hétérosis est significatif dans 2 lieux sur 3 (tableau II). Par ailleurs, pour la capacité de remobilisation, l'hybride se situe au niveau du pa- 
rent moyen (Clermont), ou entre parent moyen et parent supérieur (Le Moulon et Mons).

La relation de compensation entre assimilation post-floraison et remobilisation (Gallagher et al, 1975; Le Gouis, 1992) apparaît à Clermont et au Moulon (fig 7). Dans ces 2 lieux, cette compensation ne s'est pas faite au même niveau chez les hybrides et chez les lignées $\left(F_{2,47}=\right.$ 4,55 à Clermont: $P<0,05 ; \mathrm{F}_{2,45}=3,73$ au MouIon: $P<0,05)$. Compte-tenu des niveaux d'hétérosis observés pour $A S S$ et $R E M$, on peut en conclure que la $F_{1}$ se distingue de ses parents par une assimilation post-floraison plus importante, tout en maintenant des capacités de remobilisation équivalentes.

Le remplissage du grain peut être limité, en fonction du génotype et du milieu, aussi bien par la source que par les puits ( $\mathrm{Ma}$ et al, 1990; Blade et Baker, 1991). Nos résultats semblent indiquer que la situation hybride induit une moindre limitation au niveau de la source, tout au long de la période de remplissage des grains. En effet, une assimilation post-floraison plus importante chez la $F_{1}$ que chez ses parents, et des capacités de remobilisation équivalentes, permettent à l'hybride d'alimenter un flux d'assimilats vers les grains plus important que chez la lignée.

Cependant, il est également possible que la situation hybride soit associée à une moindre limitation par les puits. En effet, Brocklehurst (1977 et 1979) a montré que le poids d'un grain était essentiellement lié au nombre de ses cellules constitutives (et pas à la taille de ces cellules), et que le taux de division cellulaire dans le grain en formation était lié à la quantité d'assimilats disponible. Or nous avons vu qu'à la floraison les disponibilités en assimilats étaient plus importantes chez la $F_{1}$ que chez ses parents.

\section{CONCLUSION}

Notre étude multilocale montre que les blés hybrides ont des capacités de remplissage supérieures à celles des lignées, principalement en raison d'une augmentation de la vitesse de remplissage. Celle-ci apparaît liée à des disponibilités en assimilats supérieures, et donc à l'instauration de relations «source-puits" plus favorables chez l'hybride que chez la lignée. Cependant, les mécanismes physiologiques à l'origine de ce rapport "source-puits" avantageux ne sont pas connus. II serait sans doute intéressant, de ce point de vue, d'étudier la dynamique
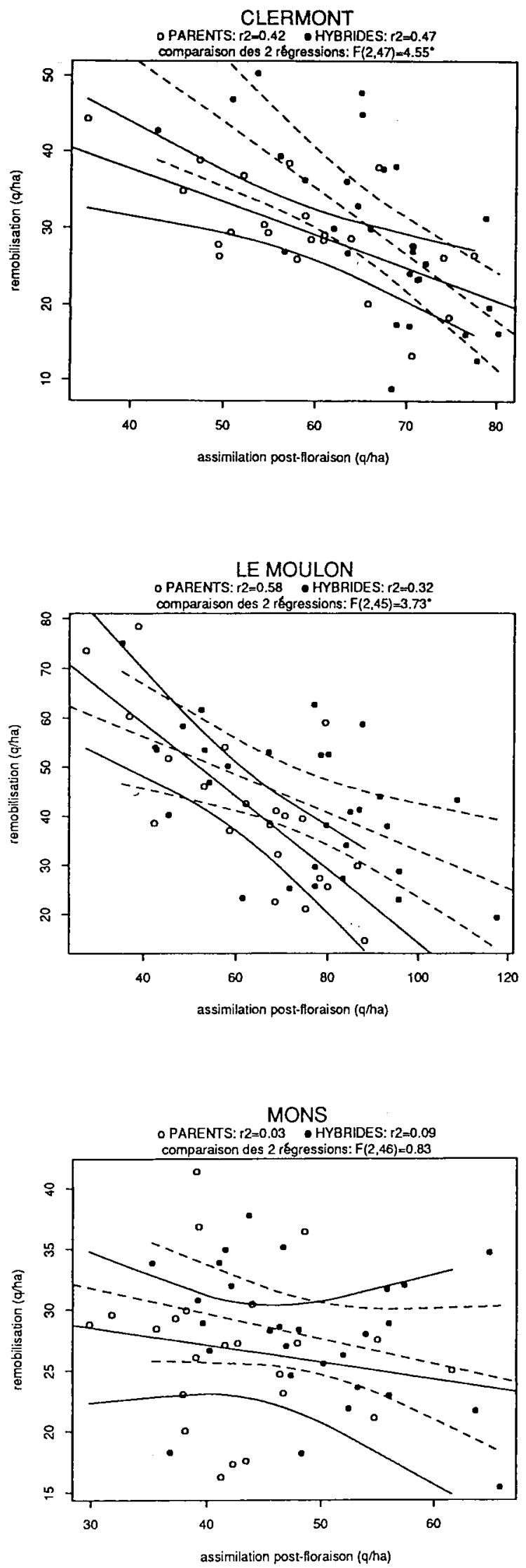

Fig 7. Relations de compensation entre ASS et REM dans les 3 lieux (la droite de régression caractérisant les hybrides est en pointillés; les courbes encadrant les droites de régressions délimitent la région de confiance à $95 \%$ de ces droites). 
des réserves carbonées et azotées chez des hybrides et leurs parents.

Par ailleurs, notre étude confirme que l'hétérosis pour le rendement est une réalité chez le blé. En effet, alors que notre échantillon de $F_{1}$ n'avait pas été choisi pour mettre en évidence les potentialités maximales des blés hybrides, le meilleur hybride a obtenu, dans les 3 lieux, un rendement au moins égal à celui de la meilleure lignée (témoins et parents confondus). C'est ainsi qu'à Clermont la meilleure $F_{1}$ est $3,3 \%$ au-dessus d'Apollo, qu'au Moulon le meilleur hybride obtient le même rendement qu'Apollo, et qu'à Mons l'hybride le plus productif est $4,4 \%$ au-dessus de la meilleure lignée (un des parents femelles). La recherche des combinaisons hybrides les plus performantes devrait donc permettre d'obtenir des $F_{1}$ supérieures aux meilleures lignées. Le problème du développement des blés hybrides se pose alors essentiellement comme un problème technico-économique de production de la semence $F_{1}$, ou plus largement de marché mondial du blé.

\section{RÉFÉRENCES}

Austin RB, Bingham J, Blackwell RD, Evans LT, Ford MA, Morgan CL, Taylor M (1980) Genetic improvements in winter wheat yields since 1900 and associated physiological changes. J Agric Sci Camb 94, 675-689.

Becker RA, Chambers JM, Wilks AR (1988) The New $S$ Language: a programming environment for data analysis and graphics. Wadsworth \& Brooks/Cole, Pacific Grove, California, 702 p

Biscoe PV, Gallagher JN, Littleton EJ, Monteith JL, Scott RK (1975) Barley and its environment IV Sources of assimilate for the grain. $J$ Appl Ecol 12, 295-318

Blade SF, Baker RJ (1991) Kernel weight response to source-sink changes in spring wheat. Crop Sci 31 , 1117-1120

Borghi B, Perenzin M, Nash RJ (1988) Agronomic and qualitative characteristics of ten bread wheat hybrids produced using a chemical hybridizing agent. Euphytica 39, 185-194

Brears T, Hydon AG, Bingham J (1988) An assessment of the feasibility of producing $F_{1}$ and $F_{2}$ hybrids for the UK. In: Proc 7th int Wheat Genet Symp, 1057-1062

Brocklehurst PA (1977) Factors controlling grain weight in wheat. Nature 266, 348-349

Brocklehurst PA (1979) Control of grain morphogenesis in wheat ans its relation to grain yield. In: Crop physiology and plant breeding. Meeting Eucarpia 1978, 41-42
Gallagher JN, Biscoe PV, Scott RK (1975) Barley and its environment $V$ Stability of grain weight. $J$ Appl Ecol 12, 319-336

Goujon C, Paquet J (1968) Contribution à l'étude de la vigueur hybride chez le blé tendre : essais de rendements en semis d'automne réalisés en 196566 et 1966-67. Ann Amélior Plant 18, 223-235

Keydel F (1973) Untersuchung über die Leistungseigenschaften von F1-Hybriden von Winterweizen. Z Pflanzenzüchtg 69, 239-255

Le Gouis J (1992) Étude de la variabilité génétique pour l'élaboration du rendement en grain de l'orge d'hiver (Hordeum vulgare $\mathrm{L}$ ). Comparaison de variétés à 2 rangs et à 6 rangs. Thèse de docteur de I'INAPG, $87 \mathrm{p}+$ annexes

Ma YZ, Mackown CT, VanSanford DA (1990) Sink manipulation in wheat: compensatory changes in kernel size. Crop Sci 30, 1099-1105

Masle-Meynard J (1980) L'élaboration du nombre d'épis chez le blé d'hiver. Influence de différentes caractéristiques de la structure du peuplement sur l'utilisation de l'azote et de la lumière. Thèse de docteur-ingénieur de l'INAPG, $201 \mathrm{p}+$ annexes

Morgan CL, Austin RB, Ford MA, Bingham J, Angus WJ, Chowdhury S (1989) An evaluation of F1 hybrid winter wheat genotypes produced using a chemical hybridizing agent. J Agric Sci Camb 112, 143-149

Oury FX, Koenig J, Bérard P, Rousset M (1990a) Une comparaison entre des blés hybrides produits par voie chimique et leurs parents : niveaux d'hétérosis et élaboration du rendement. agronomie 10, 291304

Oury F-X, Brabant P, Pluchard P, Bérard P, Rousset M (1990b) Étude multilocale de blés hybrides : niveaux d'hétérosis et élaboration du rendement. agronomie 10, 735-748

Sofield I, Evans LT, Cook MG, Wardlaw IF (1977) Factors influencing the rate and duration of grain filling in wheat. Aust J Plant Physiol 4, 785-797

Stoy $V(1980)$ Grain filling and the properties of the sink. In: Physiological aspects of crop productivity. Der Bund AG, Bern, 65-77

Tomassone R, Lesquoy E, Millier C (1983) La régression : nouveaux regards sur une ancienne méthode statistique. INRA et Masson, Paris, $180 \mathrm{p}$

Triboï $E$ (1990) Modèle d'élaboration du poids du grain chez le blé tendre (Triticum aestivum em Thell). agronomie 10, 191-200

Triboï E, Lemercier E (1985) Étude de l'interaction génotype-milieu chez le blé d'hiver: élaboration du poids du grain. In : Journées de Versailles sur l'écophysiologie du blé. Groupe "céréales à paille» de I'INRA, 161-205

Triboï E, Blanchon J, Magne J (1985) Déterminisme du poids moyen du grain chez le blé : effet sur la variation du rendement. C R Acad Agri Fr 71(8), 871-886

Triboï E, Ollier J-L (1991) Evolution et rôle des réserves glucidiques et azotées des tiges chez 21 génotypes de blé. agronomie 11, 239-246 